\title{
Measurement properties of the German version of the IKDC subjective knee form (IKDC-SKF)
}

\author{
Danica Kümmel ${ }^{1 *} \mathbb{D}$, Stefan Preiss ${ }^{2}$, Laurent P. Harder ${ }^{2}$, Michael Leunig ${ }^{2}$ and Franco M. Impellizzeri ${ }^{1}$
}

\begin{abstract}
Purpose: To examine the measurement properties of the German International Knee Documentation Committee Subjective Knee Form (IKDC-SKF) in knee disorder patients.

Methods: Three hundred twelve consecutive patients undergoing surgery for anterior cruciate ligament, meniscus and/or cartilage injuries completed the IKDC-SKF, Lysholm Score, Tegner Activity Scale, and Short Form-12 Health Survey before and 6 months post-surgery. IKDC-SKF measurement properties were calculated and patients were also asked to rate the relevance/comprehensibility of the questionnaire items.

Results: Reliability was good with high Cronbach's alpha and intraclass correlation coefficients, and standard error of measurement values of 4.4 to 6.0. The smallest detectable change (SDC) ranged from 12.3 to 16.7 points. Validity was good with $90 \%$ of all hypotheses confirmed. Confirmatory factor analysis did not show adequate fitting indices within the model. Over half of the items were rated as essential, and all were well comprehended. The majority of hypotheses for responsiveness were confirmed. No floor and ceiling effects were observed. The area under the curve ranged from 0.82 to 0.89 and the minimal important difference was smaller than the SDC.

Conclusions: The German IKDC-SKF is a reliable outcome measure with good hypotheses testing and responsiveness, but its MIC and structural/content validity need further analysis.
\end{abstract}

Keywords: IKDC, PROM, Validity, Reliability, Responsiveness

\section{Introduction}

The International Knee Documentation Committee Subjective Knee Form (IKDC-SKF) assesses symptoms, daily function and sports activity in patients with knee disorders [13, 14]. The tool has good measurement properties [9] and is freely available in several languages including German [10, 16, 19,22]. Unlike for most of the language versions available, the translation process of the German IKDC-SKF is unknown and the instrument has not been validated. There is a lack of validated German knee-specific tools to assess and compare multiple knee conditions and treatments. Within the scope of our clinical studies, a valid German IKDC-SKF

\footnotetext{
* Correspondence: danica.kuemmel@kws.ch

${ }^{1}$ Department of Teaching, Research and Development, Schulthess Clinic, Lengghalde 2, 8008 Zurich, Switzerland

Full list of author information is available at the end of the article
}

would serve as a comprehensive assessment of patients for prospective use in our clinic.

We examined the measurement properties of the German IKDC-SKF in anterior cruciate ligament (ACL), meniscus and/or cartilage injury patients undergoing surgery according to COnsensus-based Standards for the selection of health status Measurement INstruments (COSMIN) checklist [18].

\section{Materials and methods}

Patients and data collection

Seven hundred seven consecutive patients underwent ACL, meniscal and/or articular cartilage surgery in our clinic between September 2015 and December 2016. All patients completed a set of questionnaires at home within four weeks before (i.e. baseline) and 6 months after surgery. Data were excluded if items were incomplete or patients did not agree to provide 
written consent, had insufficient knowledge of the German language, did not reside in Switzerland, were under 16 years or suffered from any condition that hindered study participation. All consecutive patients who returned the questionnaire set within 12 days after completion received the IKDC-SKF again. For reliability testing, patients who completed the second IKDC-SKF questionnaire within 14 days after the first occasion were included. Psychometrics were calculated for the patient subgroups: ACL surgery only (ACL); meniscal surgery only (Meniscus); articular cartilage surgery only (Cartilage); and a combination of the three surgery types (mixed surgery subgroup = Mix).

Because the German IKDC-SKF cross-cultural adaptation is not published, we evaluated comprehensibility and relevance of each questionnaire item at baseline, 6 or 12 months post-surgery with two additional patient groups. Patients from these groups underwent surgery between April 2016 and April
2017 and were selected in further sampling procedures to increase the participation rate.

\section{Questionnaires}

IKDC-SKF examines 19 items on 5-point Likert (items 1, 4, 5, 7, 8 \& 9a-i), 0-10 rating (items 2, 3, 10a \& 10b) or dichotomous scales (item 6). The overall score is based on 18 items (item 10a is not included) and ranges from 0 to 100 points with higher points corresponding to less symptoms, better function and a higher level of sports activity [13].

The Short Form-12 (SF-12) Health Survey measures generic health status with 12 questions that are combined, scored and weighted to produce physical and mental component summary scale (PCS-12 and MCS-12) scores (0 to 100). A higher score indicates better health-related quality of life [24]. The SF-12 showed acceptable criterion validity, structural validity and reliability in ill and healthy subjects $[3,24]$.
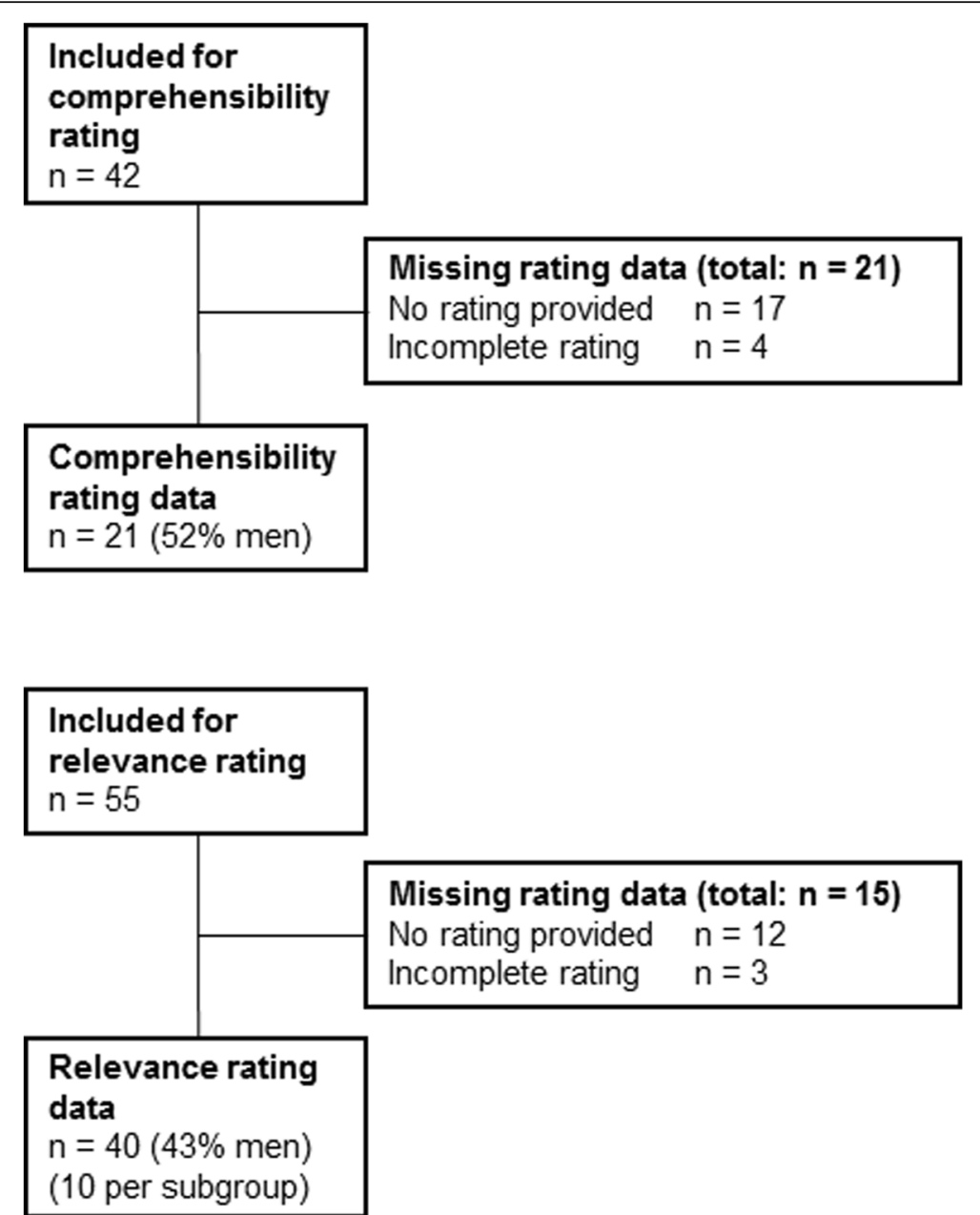

Fig. 1 Flow chart of the patient subgroup assessment of German IKDC-SKF item comprehensibility and relevance 
The Lysholm Score contains eight items measuring knee function (i.e. limping, locking, pain, stair climbing, need for support, instability, swelling and squatting) $[2,3,15]$. The total score ranges from 0 (poor) to 100 (excellent outcome without symptoms or disability). This score showed acceptable reliability, validity and responsiveness in patients with ACL/meniscal injuries and chondral disorders [2, 3, 15, 25].

The single-item Tegner Activity Scale measures the highest activity level achieved during work/sport activities [2, 3]; 0 indicates a sick leave patient and 10 indicates participation in elite-level competitive sports. This tool showed acceptable measurement properties in patients with ACL/meniscal injuries $[2,3,26]$. Cross-culturally adapted and validated German versions of all questionnaires were used [7, 25, 26].

At 6 months, patients were asked to rate their global treatment outcome (GTO) by answering: "How much did the operation help your knee problem?" on a 5-point Likert scale with the following options of (1) helped a lot; (2) helped; (3) helped only little; (4) did not help; or (5) made things worse [12].

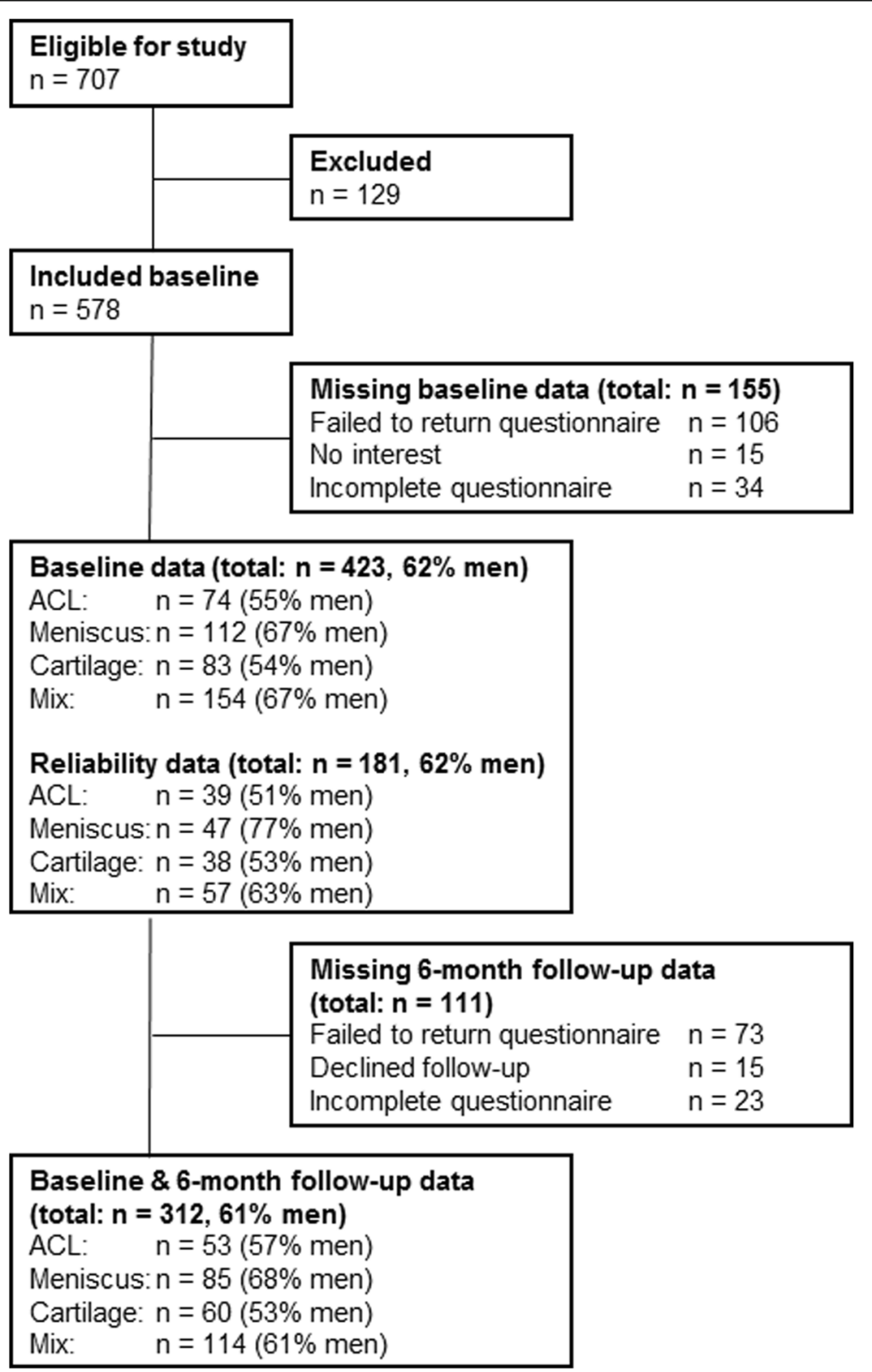

Fig. 2 Selection algorithm showing patient eligibility and the available sample sizes for assessing the measurement properties of the German IKDC-SKF 
For IKDC-SKF item comprehensibility, patients were asked, "In your opinion, how comprehensible is the question formulated?" and could answer with: (1) totally comprehensible, (2) mostly comprehensible, (3) moderately comprehensible, (4) slightly comprehensible, or (5) not at all comprehensible. In a similar manner, patients were asked to rate the relevance of each IKDC-SKF item, i.e. "In your opinion, how essential is this item in order to describe your situation?" [1] and to specify missing items, i.e. "In your opinion, which questions/items are also very important, but are missing in this questionnaire?".
We excluded incomplete questionnaire sets when more than two items were missing from the IKDC-SKF and any single item was missing from the other questionnaires.

\section{IKDC-SKF measurement properties}

Internal consistency was calculated using Cronbach's alpha with values between 0.7 and 0.95 indicating appropriate internal consistency [21]. Intraclass correlation coefficients (ICC) were calculated using a single measurement, absolute agreement, 2-way mixed-effects model to assess test-retest reliability; an ICC of at least 0.7 was

Table 1 Questionnaire scores for the total population as well as each patient subgroup

\begin{tabular}{|c|c|c|c|c|c|c|}
\hline & \multicolumn{2}{|c|}{ Examination time point } & \multicolumn{2}{|l|}{ Reliability } & \multirow[t]{2}{*}{ Comprehensibility } & \multirow[t]{2}{*}{ Relevance } \\
\hline & Baseline & 6 months & Test (T1) & Retest (T2) & & \\
\hline \multicolumn{7}{|c|}{ IKDC-SKF, mean (SD) } \\
\hline Total & $53.5(15.7)$ & $71.3(16.7)$ & $55.7(17.0)$ & $56.1(16.5)$ & \multirow[t]{5}{*}{$63.3(19.5)$} & $63.6(17.5)$ \\
\hline $\mathrm{ACL}$ & $60.2(13.5)$ & $74.2(11.5)$ & $63.3(14.1)$ & $63.8(13.2)$ & & $67.0(20.8)$ \\
\hline Meniscus & $52.2(16.6)$ & $76.7(16.8)$ & $55.7(18.6)$ & $55.0(17.4)$ & & $65.4(12.4)$ \\
\hline Cartilage & $46.8(14.4)$ & $60.6(19.5)$ & $46.8(14.5)$ & $47.8(14.1)$ & & $49.9(18.0)$ \\
\hline Mix & $54.7(15.3)$ & $71.5(14.7)$ & $56.6(16.4)$ & $57.2(17.1)$ & & $72.1(11.0)$ \\
\hline
\end{tabular}

Lysholm Score, mean (SD)

$\begin{array}{lll}\text { Total } & 61.6(17.9) & 80.5(16.4) \\ \text { ACL } & 67.7(15.2) & 82.5(14.2) \\ \text { Meniscus } & 62.0(18.3) & 84.0(15.6) \\ \text { Cartilage } & 53.7(16.7) & 69.8(18.6) \\ \text { Mix } & 62.5(18.0) & 82.8(14.6)\end{array}$

Tegner Activity Scale, mean (SD)

$\begin{array}{lll}\text { Total } & 3.2(2.0) & 3.8(1.4) \\ \text { ACL } & 3.6(2.0) & 3.9(1.5) \\ \text { Meniscus } & 3.0(1.8) & 4.0(1.5) \\ \text { Cartilage } & 2.9(1.6) & 3.2(1.5) \\ \text { Mix } & 3.4(2.3) & 3.8(1.2) \\ \text { PCS-12, mean (SD) } & & \\ \text { Total } & 41.8(10.1) & 45.2(6.6) \\ \text { ACL } & 44.0(9.6) & 45.0(6.2) \\ \text { Meniscus } & 40.7(10.2) & 46.6(5.9) \\ \text { Cartilage } & 40.3(9.0) & 41.9(7.7) \\ \text { Mix } & 42.5(10.7) & 46.0(6.2)\end{array}$

MCS-12, mean (SD)

$\begin{array}{lll}\text { Total } & 51.5(10.3) & 55.1(7.7) \\ \text { ACL } & 50.3(9.8) & 56.0(7.0) \\ \text { Meniscus } & 53.0(9.8) & 55.1(8.2) \\ \text { Cartilage } & 50.4(11.3) & 54.4(8.4) \\ \text { Mix } & 51.6(10.3) & 55.0(7.4)\end{array}$

IKDC-SKF = International Knee Documentation Committee Subjective Knee Form, PCS-12 = 12-item short-form health survey (SF-12) physical component summary scale, $M C S-12=12$-item short-form health survey (SF-12) mental component summary scale, $S D=$ standard deviation, Total = all eligible study patients, $A C L=$ anterior cruciate ligament surgery patients only, Meniscus = meniscal surgery patients only, Cartilage = articular cartilage surgery only, Mix $=$ patients from a combination of the three surgery types (mixed surgery subgroup) 
considered appropriate [21]. Agreement was assessed using the Standard Error of Measurement (SEMagreement). The smallest detectable change (SDC) was calculated using the formula: SDC $=1.96$ * $\sqrt{2}$ * SEM [5].

Item relevance was measured by counting "ne", i.e. number of patients rating an item as "essential" and comparing this value to "Ncritical" (minimum number of patients required to agree an item as "essential") [1]; Ncritical was 9 for 10 patients per subgroup and 26 for 40 patients in total. Comprehensibility was calculated by the relative frequency of patient ratings.

Structural validity was assessed by confirmatory factor analysis (CFA) using the maximum likelihood method with Satorra-Bentler adjustment. Validity was demonstrated if data fitted the recursive one factor structure proposed in a number of studies $[9,13,20,22]$ with or without item 6 as part of the model. CFA was also performed without item 6 in order to achieve a better model fit, since item 6 is dichotomous and had low factor loadings in previously performed analyses [13, 20, 22]. Validity was good if at least $75 \%$ of the tested hypotheses were confirmed [21]. Strong correlations overall and for each subgroup $(\geq 0.6)$ were expected between the IKDC-SKF and SF-12 (PCS-12) as well as IKDC-SKF and Lysholm Score $[4,13,16,23]$. Weak to moderate correlations (0.1-0.49) were expected between the IKDC-SKF and Tegner Activity Scale $[3,6]$. Furthermore, we expected correlations of $0.1-0.29$ for the IKDC-SKF and SF-12 (MCS-12) [13, 16].

Responsiveness was assessed with predefined hypotheses on the change scores between baseline and 6 months. Moderate correlations $(\geq 0.4)$ for the entire population and each subgroup were expected between the change scores of the IKDC-SKF and SF-12 (PCS-12), and IKDC-SKF and Lysholm Score [22, 23]. A strong, inverse correlation $(\leq-0.6)$ was expected between the change scores of the IKDC-SKF and GTO.

In general, the effect size (ES) and standardised response mean (SRM) were expected to be large $(\geq 0.8)[4,14,15]$.
Floor and ceiling effects were considered absent if percentages were below 15\% [21]. To assess minimal important change (MIC), receiver operating characteristics (ROC) were analysed with the GTO as the anchor question. Patients who stated that the operation helped or helped a lot were considered to have a good outcome; all other responses indicated a poor outcome. The MIC was estimated as the optimal ROC cut-off point represented by the smallest value equivalent to the sum of 1-sensitivity and 1-specificity [5]. Area under the curve (AUC) was calculated as a measure of discriminant ability.

All analyses were performed using Stata Corp. 2015 Stata Statistical Software: Release 14 (StataCorp LP, College Station, TX, USA).

\section{Results}

The number of patients for assessing content validity and all measurement properties are shown in Figs. 1 and 2, respectively. The mean age of the patient population was 38 years (SD 14 years; range 16-74 years). Mean IKDC-SKF scores at baseline and 6 months were 53.5 and 71.3, respectively (Table 1 ).

For the entire patient cohort, Cronbach's alpha was 0.87 ; similar values were calculated for the various subgroups (0.84-0.89).

ICC was 0.94 (95\%CI, 0.91-0.95) for the total cohort and similar for ACL, Meniscus, Cartilage and Mix subgroups. SEMagreement values were also similar among the patient groups (range 4.4-6.0 points) as were the SDC values (range 12.3-16.7 points) (Table 2).

IKDC-SKF items were rated as totally or mostly comprehensible by the majority $(\geq 86 \%)$ of patients. Some items were also rated as slightly or not comprehensible by $4.8 \%$ (item $1,3,7,9 \mathrm{~d}, 9 \mathrm{i}, 10 \mathrm{a}$ ) and $15.3 \%$ (item 2 ) of patients. In the total patient sample, $52.6 \%$ of items were rated "essential" by a sufficient number of patients (ne $\geq 26$ ). With a ne value $\geq 9$, the following percentage of items were rated as essential by the ACL, Cartilage and

Table 2 IKDC-SKF reliability and minimal important change

\begin{tabular}{llllll}
\hline & \multicolumn{1}{l}{ IKDC-SKF } & & & \\
\cline { 2 - 6 } & Total & ACL & Meniscus & Cartilage & Mix \\
\hline Cronbach's Alpha $^{\mathrm{a}}(95 \% \mathrm{Cl})$ & $0.87(0.85 ; 0.89)$ & $0.84(0.76 ; 0.92)$ & $0.89(0.86 ; 0.92)$ & $0.87(0.83 ; 0.91)$ & $0.87(0.84 ; 0.90)$ \\
ICC $^{\text {b }}(95 \% \mathrm{Cl})$ & $0.94(0.91 ; 0.95)$ & $0.93(0.86 ; 0.96)$ & $0.93(0.89 ; 0.96)$ & $0.92(0.85 ; 0.96)$ & $0.93(0.88 ; 0.96)$ \\
SEM $^{\mathrm{b}}$ & 5.4 & 4.4 & 5.6 & 6.0 & 5.5 \\
SDC $^{\mathrm{b}}$ & 14.9 & 12.3 & 15.6 & 16.7 & 15.3 \\
MIC $^{\mathrm{C}}$ & 6.7 & -1.3 & 11.3 & 12.5 & 12.5 \\
\hline
\end{tabular}

$I C C=$ intraclass correlation coefficient, $C l=$ confidence interval, $S E M=$ standard error of measurement, $S D C=$ smallest detectable change, $M I C=$ minimal important change, IKDC-SKF = International Knee Documentation Committee Subjective Knee Form, Total = all eligible study patients, $A C L=$ anterior cruciate ligament surgery patients only, Meniscus = meniscal surgery patients only, Cartilage = articular cartilage surgery only, Mix = patients from a combination of the three surgery types (mixed surgery subgroup)

${ }^{a}$ Sample sizes for Cronbach's alpha calculations are equivalent to baseline data " $n$ " in Fig. 2

bSample sizes for ICC, SEM and SDC calculations are equivalent to reliability data " $\mathrm{n}$ " in Fig. 2

"Sample sizes for the MIC calculation are equivalent to baseline \& 6-month follow-up data " $\mathrm{n}$ " in Fig. 2 
Table 3 Hypothesis testing for evaluating validity of the IKDC-SKF for the total population as well as each patient subgroup

\begin{tabular}{llllll}
\hline & IKDC-SKF & & & & \\
\cline { 2 - 6 } & Total & ACL & Meniscus & Cartilage & Mix \\
\hline Lysholm & $\mathbf{0 . 8 0}(\geq 0.6)$ & $\mathbf{0 . 7 5}(\geq 0.6)$ & $\mathbf{0 . 7 9}(\geq 0.6)$ & $\mathbf{0 . 8 1}(\geq 0.6)$ & $\mathbf{0 . 8}(\geq 0.6)$ \\
Tegner & $\mathbf{0 . 3 4}(0.1-0.49)$ & $\mathbf{0 . 3 9}(0.1-0.49)$ & $\mathbf{0 . 3 7}(0.1-0.49)$ & $\mathbf{0 . 4 2}(0.1-0.49)$ & $\mathbf{0 . 2 5}(0.1-0.49)$ \\
PCS-12 & $\mathbf{0 . 7 0}(\geq 0.6)$ & $\mathbf{0 . 7 1}(\geq 0.6)$ & $\mathbf{0 . 6 7}(\geq 0.6)$ & $\mathbf{0 . 6 8}(\geq 0.6)$ & $\mathbf{0 . 7 2}(\geq 0.6)$ \\
MCS-12 & $\mathbf{0 . 1 7}(0.1-0.29)$ & $\mathbf{0 . 1 3}(0.1-0.29)$ & $\mathbf{0 . 2 3}(0.1-0.29)$ & $0.30(0.1-0.29)$ & $0.09(0.1-0.29)$ \\
\hline
\end{tabular}

Correlation estimates in parentheses indicate a priori formulated hypotheses

Correlation estimates in bold indicate confirmed hypotheses

IKDC-SKF = International Knee Documentation Committee Subjective Knee Form, Lysholm = Lysholm Score, Tegner $=$ Tegner Activity Scale, $P C S$ - $12=12$-item shortform health survey (SF-12) physical component summary scale, MCS-12 = 12-item short-form health survey (SF-12) mental component summary scale, Total = all eligible study patients, $A C L=$ anterior cruciate ligament surgery patients only, Meniscus = meniscal surgery patients only, Cartilage = articular cartilage surgery only, Mix = patients from a combination of the three surgery types (mixed surgery subgroup)

Mix subgroups: 5.3\% (item 7); 36.8\% (items 1, 2, 5, 8, 9i, 10a \& b); and 5.3\% (items $9 \mathrm{~d} \& \mathrm{~h}$ ), respectively; none of the items were essential for the Meniscus group. Eight patients suggested additional items covering the following topics: ACL: pain quality; Meniscus: avoided sport activities; Cartilage: previous surgery, inability to work; employment Mix: possible impulsive movements, strength training, previous knee surgery.

The lowest factor loading for item 6 was 0.15 , and over 0.4 for all other items. Satorra-Bentler adjusted goodness of fit parameters were inadequate when taking item 6 into consideration $\left(x^{2}(\mathrm{df})\right.$ (chi-square statistic [degrees of freedom]): 1101.84 (135), p: 0.00; Comparative Fit Index (CFI): 0.696; Tucker-Lewis index (TLI): 0.655 ; root mean squared error of approximation (RMSEA): 0.131 ; standardised root mean squared residual (SRMR): 0.096; coefficient of determination (CD): $0.918)$ or not ( $X^{2}(\mathrm{df}): 1086.74(119)$, p: 0.00; CFI: 0.696; TLI:0.652; RMSEA: 0.139; SRMR 0.1; CD 0.918). The majority of hypotheses evaluating construct validity (90\%) (Table 3) and responsiveness (76\%) were confirmed (Table 4).

No floor and ceiling effects were observed. AUC values ranging from 0.82 to 0.89 for all groups were considered appropriate, but calculated MICs varied considerably (total [6.8], ACL [-1.3], Meniscus [11.3], Cartilage [12.5], and Mix [6.7]).

\section{Discussion}

Our study showed good reliability and hypotheses testing of the IKDC-SKF, which is in line with previous investigations $[4,9,10,13,15-17,19,22,23]$.

CFA revealed similar factor loadings compared to other studies [13, 20, 22]. Nevertheless, our data do not fit the hypothesised model based on the fit indices. The application of uni- and two-dimensional scales for the IKDC-SKF is debatable $[9,11,13,20,22]$. This indicates that the resulting lack of structural validity can be attributed to the construct rather than the translation.

Over half of the items were rated as essential by at least $65 \%$ of patients in the total population, and up to almost $40 \%$ of the items were rated as essential by a minimum of $90 \%$ of patients in the subgroups.

The variation between subgroups may indicate different priorities, various main symptoms and that shorter questionnaires are preferred; the additional items suggested by our patients seem to support the differences in their concerns and symptoms. All items were rated as comprehensible, and we believe the general wording is sufficient. We consider content validity to be

Table 4 Responsiveness of the IKDC-SKF for the total population as well as each patient subgroup

\begin{tabular}{|c|c|c|c|c|c|}
\hline & IKDC-SKF & & & & \\
\hline & $\Delta$ total & $\triangle A C L$ & $\Delta$ Meniscus & $\Delta$ Cartilage & $\Delta \operatorname{Mix}$ \\
\hline$\Delta$ Lysholm & $\mathbf{0 . 7 4}(\geq 0.4)$ & $0.69(\geq 0.4)$ & $\mathbf{0 . 7 9}(\geq 0.4)$ & $\mathbf{0 . 7 5}(\geq 0.4)$ & $\mathbf{0 . 7 2}(\geq 0.4)$ \\
\hline$\Delta \mathrm{PCS}-12$ & $0.60(\geq 0.4)$ & $0.57(\geq 0.4)$ & $\mathbf{0 . 5 4}(\geq 0.4)$ & $\mathbf{0 . 6 8}(\geq 0.4)$ & $\mathbf{0 . 5 9}(\geq 0.4)$ \\
\hline GTO & $-0.55(\leq-0.6)$ & $-0.45(\leq-0.6)$ & $-0.56(\leq-0.6)$ & $-0.66(\leq-0.6)$ & $-0.51(\leq-0.6)$ \\
\hline ES & $1.04(\geq 0.8)$ & $1.11(\geq 0.8)$ & $1.39(\geq 0.8)$ & $0.75(\geq 0.8)$ & $1.06(\geq 0.8)$ \\
\hline SRM & $\mathbf{0 . 9 6}(\geq 0.8)$ & $\mathbf{0 . 8 4}(\geq 0.8)$ & $1.23(\geq 0.8)$ & $0.71(\geq 0.8)$ & $0.99(\geq 0.8)$ \\
\hline
\end{tabular}

Correlation estimates in parentheses indicate a priori formulated hypotheses

Correlation estimates in bold indicate confirmed hypotheses

$\Delta=$ change score

IKDC-SKF = International Knee Documentation Committee Subjective Knee Form, $P C S-12=12$-item short-form health survey (SF-12) physical component summary scale, $G T O=$ global treatment outcome, $E S=$ effect size, $S R M=$ standardised response mean, $T$ otal = all eligible study patients, $A C L=$ anterior $c r u c i a t e$ ligament surgery patients only, Meniscus = meniscal surgery patients only, Cartilage $=$ articular cartilage surgery only, Mix $=$ patients from a combination of the three surgery types (mixed surgery subgroup) 
moderate. A comparison to previous studies is difficult, since evaluation of patient opinion on item relevance has been addressed in few studies including ACL patients and different analytical methods, and item comprehensiveness is lacking [22, 23].

The German IKDC-SKF showed good responsiveness and no floor and ceiling effects; these characteristics are confirmed in previous studies [8, 9, 14, 22, 23]. MIC was smaller than SDC for all test groups, which indicates a suboptimal ability to distinguish clinically relevant changes from measurement error at the individual level. However, this finding is in line with previous studies comparing SDC with MIC at 4 and 6 months post-surgery $[8,22]$.

A potential limitation could be our GTO, which was applied as the anchor for MIC calculation and refers to a change based on the surgery alone. Since all patients received standard clinical treatment, we believe this GTO can be used to satisfactorily assess change.

\section{Conclusion}

The German IKDC-SKF is a reliable tool showing good hypotheses testing and responsiveness for patients with $\mathrm{ACL}$, meniscus and/or cartilage disorders undergoing surgery. However, structural/content validity and MIC require further analysis.

\section{Acknowledgements}

The authors would like to thank Laura Duc, Selina Nauer, Myrta Rüegger and Anika Stephan for their valuable assistance with the data collection as well as Dr. Melissa Wilhelmi for her support in preparing the manuscript.

\section{Ethical approval}

All procedures performed in studies involving human participants were in accordance with the ethical standards of the institutional and/or national research committee and with the 1964 Helsinki declaration and its later amendments or comparable ethical standards. This study was approved by the ethics committee of the Canton Zurich (PB_2017-000307).

\section{Availability of data and materials}

Data will not be shared. All subjects agreed to the use of their data for research purposes. However, some subjects refused the transfer of their data to third parties.

\section{Authors' contributions}

$\mathrm{DK}, \mathrm{SP}, \mathrm{LPH}, \mathrm{ML}$ and FMI contributed to the conception and design of the study. DK conducted data analysis. DK and FMl interpreted data. DK drafted the first version of the manuscript and SP, LPH, ML and FMl revised the work critically for important intellectual content. All authors read and approved the final manuscript.

\section{Authors' information}

DK': MA, research assistant.

$S P^{2}: M D$, departmental head knee surgery.

$\mathrm{LPH}^{2}: \mathrm{MD}$, senior consultant.

$\mathrm{ML}^{1}$ : $\mathrm{PD}, \mathrm{MD}$, clinical head of research lower extremities, departmental head hip surgery.

$\mathrm{FMI}^{2}: \mathrm{PhD}$, head of research group lower extremities.

\section{Consent for publication}

Informed consent was obtained from all individual participants included in the study.

\section{Competing interests}

M. L. reports having received personal fees from Smith \& Nephew, Biomed and Pivot, outside the submitted work. All other authors declare that they have no competing interest.

\section{Publisher's Note}

Springer Nature remains neutral with regard to jurisdictional claims in published maps and institutional affiliations.

\section{Author details}

'Department of Teaching, Research and Development, Schulthess Clinic, Lengghalde 2, 8008 Zurich, Switzerland. ${ }^{2}$ Musculoskeletal Centre, Orthopaedics Lower Extremities, Schulthess Clinic, Lengghalde 2, 8008 Zurich, Switzerland.

Received: 14 December 2017 Accepted: 3 July 2018

Published online: 13 July 2018

\section{References}

1. Ayre, C., \& Scally, A. J. (2013). Critical values for Lawshe's content validity ratio: Revisiting the original methods of calculation. Measurement and Evaluation in Counseling and Development, 47(1), 79-86. https://doi.org/10. $1177 / 0748175613513808$

2. Briggs, K. K., Kocher, M. S., Rodkey, W. G., \& Steadman, J. R. (2006). Reliability, validity, and responsiveness of the Lysholm knee score and Tegner activity scale for patients with meniscal injury of the knee. The Journal of Bone and Joint Surgery. American Volume, 88(4), 698-705. https://doi.org/10.2106/JBJS. E.00339.

3. Briggs, K. K., Lysholm, J., Tegner, Y., Rodkey, W. G., Kocher, M. S., \& Steadman, J. R. (2009). The reliability, validity, and responsiveness of the Lysholm score and Tegner activity scale for anterior cruciate ligament injuries of the knee: 25 years later. The American Journal of Sports Medicine, 37(5), 890-897. https://doi.org/10.1177/0363546508330143.

4. Crawford, K., Briggs, K. K., Rodkey, W. G., \& Steadman, J. R. (2007). Reliability, validity, and responsiveness of the IKDC score for meniscus injuries of the knee. Arthroscopy, 23(8), 839-844. https://doi.org/10.1016/ j.arthro.2007.02.005

5. De Vet, H. C., Terwee, C. B., Mokkink, L. B., \& Knol, D. L. (2011). Measurement in medicine: a practical quide. Cambridge: Cambridge University Press.

6. Eshuis, R., Lentjes, G. W., Tegner, Y., Wolterbeek, N., \& Veen, M. R. (2016). Dutch translation and cross-cultural adaptation of the Lysholm score and Tegner activity scale for patients with anterior cruciate ligament injuries. The Journal of Orthopaedic and Sports Physical Therapy, 46(11), 976-983. https://doi.org/10.2519/jospt.2016.6566.

7. Gandek, B., Ware, J. E., Aaronson, N. K., Apolone, G., Bjorner, J. B., Brazier, J. E., et al. (1998). Cross-validation of item selection and scoring for the SF-12 health survey in nine countries: results from the IQOLA project International quality of life assessment. Journal of Clinical Epidemiology, 51(11), 1171-1178.

8. Greco, N. J., Anderson, A. F., Mann, B. J., Cole, B. J., Farr, J., Nissen, C. W., et al. (2010). Responsiveness of the international knee documentation committee subjective knee form in comparison to the western Ontario and McMaster universities osteoarthritis index, modified Cincinnati knee rating system, and short form 36 in patients with focal articular cartilage defects. The American Journal of Sports Medicine, 38(5), 891-902. https://doi.org/10.1177/ 0363546509354163

9. Grevnerts, H. T., Terwee, C. B., \& Kvist, J. (2014). The measurement properties of the IKDC-subjective knee form. Knee Surgery, Sports Traumatology, Arthroscopy. https://doi.org/10.1007/s00167-014-3283-z.

10. Haverkamp, D., Sierevelt, I. N., Breugem, S. J., Lohuis, K., Blankevoort, L., \& van Dijk, C. N. (2006). Translation and validation of the Dutch version of the international knee documentation committee subjective knee form. The American Journal of Sports Medicine, 34(10), 1680-1684. https://doi.org/10. 1177/0363546506288854

11. Higgins, L. D., Taylor, M. K., Park, D., Ghodadra, N., Marchant, M., Pietrobon, R, et al. (2007). Reliability and validity of the international knee documentation committee (IKDC) subjective knee form. Joint, Bone, Spine, 74(6), 594-599. https://doi.org/10.1016/j.jbspin.2007.01.036.

12. Impellizzeri, F. M., Leunig, M., Preiss, S., Guggi, T., \& Mannion, A. F. (2017) The use of the core outcome measures index (COMI) in patients undergoing total knee replacement. Knee, 24(2), 372-379. https://doi.org/10. 1016/j.knee.2016.11.016. 
13. Irrgang, J. J., Anderson, A. F., Boland, A. L., Harner, C. D., Kurosaka, M., Neyret, P., et al. (2001). Development and validation of the international knee documentation committee subjective knee form. The American Journal of Sports Medicine, 29(5), 600-613.

14. Irrgang, J. J., Anderson, A. F., Boland, A. L., Harner, C. D., Neyret, P., Richmond, J. C., et al. (2006). Responsiveness of the international knee documentation committee subjective knee form. The American Journal of Sports Medicine, 34(10), 1567-1573. https://doi.org/10.1177/ 0363546506288855.

15. Kocher, M. S., Steadman, J. R., Briggs, K. K., Sterett, W. I., \& Hawkins, R. J. (2004). Reliability, validity, and responsiveness of the Lysholm knee scale for various chondral disorders of the knee. The Journal of Bone and Joint Surgery. American Volume, 86-a(6), 1139-1145.

16. Metsavaht, L., Leporace, G., Riberto, M., de Mello Sposito, M. M., \& Batista, L. A. (2010). Translation and cross-cultural adaptation of the Brazilian version of the international knee documentation committee subjective knee form: Validity and reproducibility. The American Journal of Sports Medicine, 38(9), 1894-1899. https://doi.org/10.1177/0363546510365314.

17. Metsavaht, L., Leporace, G., Riberto, M., Sposito, M. M., Del Castillo, L. N., Oliveira, L. P., et al. (2012). Translation and cross-cultural adaptation of the lower extremity functional scale into a Brazilian Portuguese version and validation on patients with knee injuries. The Journal of Orthopaedic and Sports Physical Therapy, 42(11), 932-939. https://doi.org/ 10.2519/jospt.2012.4101.

18. Mokkink, L. B., Terwee, C. B., Knol, D. L., Stratford, P. W., Patrick, D. L., Bouter, L. M., \& de Vet, H. C. W. (2010). The COSMIN checklist for evaluating the methodological quality of studies on measurement properties: A clarification of its content. BMC Medical Research Methodology, 10(1), 22. https://doi.org/10.1186/1471-2288-10-22.

19. Padua, R., Bondi, R., Ceccarelli, E., Bondi, L., Romanini, E., Zanoli, G., et al. (2004). Italian version of the international knee documentation committee subjective knee form: Cross-cultural adaptation and validation. Arthroscopy, 20(8), 819-823. https://doi.org/10.1016/j.arthro.2004.06.011.

20. Schmitt, L. C., Paterno, M. V., \& Huang, S. (2010). Validity and internal consistency of the international knee documentation committee subjective knee evaluation form in children and adolescents. The American Journal of Sports Medicine, 38(12), 2443-2447. https://doi.org/10.1177/ 0363546510374873.

21. Terwee, C. B., Bot, S. D., de Boer, M. R., van der Windt, D. A., Knol, D. L. Dekker, J., et al. (2007). Quality criteria were proposed for measurement properties of health status questionnaires. Journal of Clinical Epidemiology, 60(1), 34-42. https://doi.org/10.1016/j.jclinepi.2006.03.012.

22. Tigerstrand Grevnerts, H., Grävare Silbernagel, K., Sonesson, S., Ardern, C., Österberg, A., Gauffin, H., et al. (2017). Translation and testing of measurement properties of the Swedish version of the IKDC subjective knee form. Scandinavian Journal of Medicine \& Science in Sports, 27(5), 554-562. https://doi.org/10.1111/sms.12861.

23. van Meer, B. L., Meuffels, D. E., Vissers, M. M., Bierma-Zeinstra, S. M., Verhaar J. A., Terwee, C. B., et al. (2013). Knee injury and osteoarthritis outcome score or international knee documentation committee subjective knee form: Which questionnaire is most useful to monitor patients with an anterior cruciate ligament rupture in the short term? Arthroscopy, 29(4), 701-715. https://doi.org/10.1016/j.arthro.2012.12.015.

24. Ware, J. E., Keller, S. D., \& Kosinski, M. (1995). SF-12: How to score the SF-12 physcial and mental health summary scales (2nd ed.). Boston: The Health Institute, New England Medical Center.

25. Wirth, B., Liffert, F., \& de Bruin, E. D. (2011). Development and evaluation of a German version of the Lysholm score for measuring outcome after anterior cruciate ligament injuries. Sportverletzung Sportschaden, 25(1), 37-43. https://doi.org/10.1055/s-0029-1245825.

26. Wirth, B., Meier, N., Koch, P. P. \& Swanenburg, J. (2013). Development and evaluation of a German version of the Tegner activity scale for measuring outcome after anterior cruciate ligament injury. Sportverletzung Sportschaden, 27(1), 21-27. https://doi.org/10.1055/s-0032-1330752. 